\title{
Hierarchical fuzzy-based support system for anaesthesia monitoring and control
}

\author{
J.S. Shieh, M.F. Abbod, D.A. Linkens and J.E. Peacock

\begin{abstract}
The paper describes research into a novel hierarchical architecture for fuzzy logic monitoring and control of intravenous anaesthesia with two main objectives. The primary task is to utilise auditory evoked response signals for augmenting cardiovascular and body function signs into a multisensor fuzzy model-based fusion strategy for anaesthesia monitoring and control. The secondary task is to extend an existing fuzzy patient model for use as a training simulator via computer-based models. This demonstrates a totally fuzzy-logic-based architecture for manipulating complex procedures on a complex process (i.e. hospital patients).
\end{abstract}

$\begin{array}{ll}\text { Notation } & \\ \text { JEG } & \text { Electroencephalogram } \\ \text { AER } & \text { Audio evoked response } \\ \text { MLAER } & \text { Mid latency AER } \\ \text { SAP } & \text { Systolic arterial pressure } \\ \text { HR } & \text { Heart rate } \\ \text { \$W } & \text { Sweating } \\ \text { IA } & \text { Lacrimation } \\ \text { JR } & \text { Pupil response } \\ \text { DOA } & \text { Depth of anaesthesia } \\ \text { PDOA } & \text { Primary level of DOA } \\ \text { DDOA } & \text { Dominant level of DOA } \\ \text { FDOA } & \text { First level of DOA } \\ \text { SDOA } & \text { Secondary level of DOA } \\ \text { DOL } & \text { Depth of lightness } \\ \text { CDOA } & \text { Confidence in DOA } \\ \text { AL } & \text { Anaesthetic light } \\ \text { AO } & \text { Anaesthetic OK } \\ \text { AD } & \text { Anaesthetic deep } \\ \text { \$OFLC } & \text { Self-organising fuzzy logic controller } \\ \text { \$OFM } & \text { Self-organising fuzzy model } \\ \text { LC } & \text { Linguistic controller } \\ \text { TCI } & \text { Target controlled infusion } \\ \text { IR } & \text { Infusion rate }\end{array}$

\section{Introduction}

Motivated by hierarchical structures in complex industrial systems and in management systems, theoretical investiga-

\section{(i) IEE, 1999}

IFE Proceedings online no. 19990125

LOI: $10.1049 / \mathrm{ip}-\mathrm{cta}: 19990125$

Paper first received 2nd December 1998

J.S. Shieh is with the Center for Biomedical Engineering, National Taiwan University, Taipei, Taiwan, Republic of China

E-mail: jsshieh@anesthesia.mc.ntu.edu.tw.

A.F. Abbod and D.A. Linkens are with the Department of Automatic C'ontrol and Systems Engineering, University of Sheffield, Mappin Street, Sheffield S13 3JD, UK

E.-mail: D.Linkens@sheffield.ac.uk

J.E. Peacock is with the Department of Anaesthesia, Royal Hallamshire HLospital, Sheffield, UK

IfE Proc.-Control Theory Appl., Vol. 146, No. 3, May 1999 tions in establishing the decomposition and co-ordination approach for large-scale systems were explored by Mesarovic et al. [1]. The development of so-called hierarchical control has grown significantly in the past two decades in industries such as steel [2], petrochemistry [3] and electric power [4-6]. Through multilevel methodologies, a largescale control system's complexity can be reduced by solving a family of subproblems which are of smaller dimensions and are more easily handled. Hierarchical control has been extended to large-scale control systems with multiple objectives [7-9]. Furthermore, intelligent multiobjective optimal control has been successfully applied to control a train transportation process using hierarchical intelligent control combined with fuzzy logic [10].

Within the current interest in intelligent systems engineering, one of the major paradigms being emphasised is that of fuzzy logic control. Although fuzzy theory was invented by Zadeh in 1965 [11], and fuzzy control was demonstrated by Mamdani in 1974 [12], there occurred a delay of two decades before the commercial importance of this technique was to be demonstrated by Japanese products and latterly by German industry. Thus, now there are commercial and industrial fuzzy logic based products covering a vast range of high-volume, low-cost, low-technology consumer products through to high-technology aerospace applications [13, 14].

Some of the main advantages of fuzzy control are that it is good for nonlinear systems, or where mathematical models are either unobtainable or too complex, or where there is large uncertainty about process (e.g. hospital patient) parameters. Obviously, the field of biomedicine ought to be a natural candidate for these situations. Surprisingly, therefore, the use of fuzzy control has been slow to mature in clinical engineering. While there are several uses of fuzzy logic for signal processing and interpretation in medicine, there are relatively few examples of fuzzy control. In 1988, one of the authors published an early study on fuzzy control of muscle relaxation, via simulation [15]. More recently, this has been implemented in extensive clinical trials. Fuzzy control has been applied to blood pressure control on humans in the USA [16]. Recently, several researchers [17-19] have constructed closed-loop blood pressure control systems based on fuzzy logic during isoflurane or enflurane anaesthesia. 
The results show the fuzzy controller to be able not only to imitate the anaesthetist's management of the administration of the inhaled gas but also to give superior performance. However, an argument heard frequently against this practice is that blood pressure depends on many factors such as blood volume and cardiovascular function, apart from anaesthetic depth.

Depth of anaesthesia (DOA) is a very challenging area for fuzzy control since direct measurements are unavailable. Thus, a hierarchical structure containing fuzzy reasoning at every level has been developed [20]. This provides self-organising fuzzy modelling (SOFM) at the first level for inferential monitoring of anaesthetic depth utilising numerical clinical variables, such as systolic arterial pressure (SAP) and heart rate (HR). The second level is focused on non-numerical clinical signs, such as sweating (SW), lacrimation (LA) and pupil response (PR), which can be merged with the first level to decide DOA with more confidence. At the next level, regulatory control is achieved for the anaesthesia maintenance phase via a self-organising fuzzy logic controller (SOFLC). Hence, the existing fuzzy logic based programme provides good control algorithms with self-learning capabilities, but only limited measurement inference which is based mostly on cardiovascular indicators (i.e. changes in SAP and HR). However, these changes are subject to moderation by drugs used in anaesthesia (e.g. opioids and anticholinergics), by the surgical situation (e.g. posture) or patients' circumstances (e.g. fluid loss and blood loss), and hence they are unreliable in practice.

In the search for a more reliable monitor of DOA the electroencephalogram (EEG) is an obvious signal to investigate. The EEG, generated from within the central nervous system (CNS), is not affected by neuromuscular blockers, and the raw signal has been known for some time to show graded changes with increasing concentration of anaesthetic agents. Numerous drugs and signal processing techniques have been investigated to find a widely acceptable index of DOA. Thus, Buhrer et al. [21] used fast Fourier transforms and a periodic time series analysis to study the effect of the drug midazolam on the central nervous system. They concluded that the EEG signal was best classified via the use of periodic time analysis parameters. Stanski [22] states that the changes in morphology due to anaesthetic agents are profound and also different for each class of drug. For thiopental, with an increasing concentration in the plasma, initially the frequency and amplitude of EEG rhythms increases. In stage 2 there is a decrease in frequency and increase in amplitude, while stage 3 produces burst suppression, followed by an isoelectric condition in stage 4 .

Frequency-based features for classification have included spectral edge (frequency below which $95 \%$ of the EEG power exists) or the median frequency (50th percentage of EEG power). Hoffman and Edelman [23] showed that burst suppression, quantified via percentage times of electrical silence, is similar for inhalational agents isoflurane and desflurane used in neurosurgery. The complexity of the EEG component rhythms has led people to investigate higher-order moments in the frequency analysis, in attempts to elicit information about interactions between the rhythms. Thus, Barnett et al. [24] described the use of the bispectrum for distinguishing between awake and sleeping persons. More recently, the bispectral index has been developed into commercially available monitors of DOA. The inherent complexity of the signal generators in the brain responsible for the spontaneous EEG make it difficult to propose a widely acceptable index of DOA.
Attention has turned therefore to evoked potentials (EP). EPs, which are changes in electrical potential evoked by auditory, somatosensory or visual stimuli, have two advantages over the EEG in the study of anaesthesia: EP is an indication of the responsiveness of the CNS, whereas the EEG reflects the resting level, and EPs have anatomical significance. The auditory evoked response (AER) has been tested with these criteria in mind [25]. Thus, having been validated as a measure of DOA the mid-latency auditory evoked response (MLAER) [26], is being used in the clinical context. The main features of MLAER, which reflect the anaesthetic depth, are the changes in latencies and amplitudes of its waves due to drug step changes and surgical stimuli. Digital signal processing techniques have been carried out to smooth MLAER and improve the signal-to-noise ratio. Feature extraction has been implemented to extract the factors describing the changes in amplitudes and latencies of MLAER waves which are related to DOA. Thus, three factors have been obtained and fused together using fuzzy logic to create a reliable index for DOA [27].

The overall purpose of this paper is to consolidate and extend research into a novel hierarchical architecture for fuzzy logic monitoring and control of intravenous anaesthesia, with two main objectives. The primary task is to utilise AER signals for augmenting cardiovascular and body function signs into a multisensor fuzzy modelbased fusion strategy for anaesthesia monitoring and control. The secondary task is to extend an existing fuzzy patient model for use as a training simulator via realistic computer-based models. This will then demonstrate a totally fuzzy logic based architecture for manipulating complex procedures on a complex process (i.e. hospital patients)

\section{Hierarchical structure for monitoring DOA}

A hierarchical structure using cardiovascular indicators. AER signals and clinical signs to monitor DOA is shown in Fig. 1. The first level uses measured data which can be input online to the system, such as SAP, HR and AER. The second level focuses on clinical signs which are nonnumerical and difficult to apply online to the system via

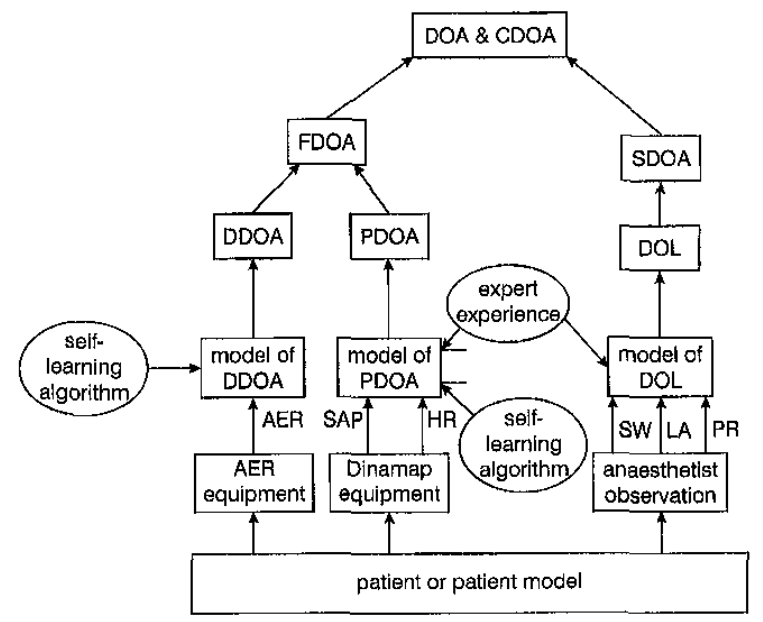

Fig. 1 Hierarchical structure for monitoring $D O A$ For notation see beginning of paper 
a computer. These clinical signs, such as SW, LA and PR, need to be input to the computer manually after observation by the anaesthetist. After the data from the first and second levels have been fused, the DOA can be decided more accurately.

\subsection{First level of depth of anaesthesia (FDOA) from SAP, HR and AER}

The first level estimates the primary depth of anaesthesiology (PDOA) from online signals such as SAP and HR, as shown in Fig. 1. SAP and HR are divided into three different ranges i.e. high, medium and low. High means the SAP and HR values of the patient are higher than pormal values (patient-specific) and vice versa for low. Medium means the SAP and HR values of the patient are in the normal range. There are also three states of anaesthesia i.e. anaesthetic light (AL), anaesthetic $O K(\mathrm{AO})$ and anaesthetic deep (AD). At this level, the rules can come from anaesthetists' experience, in which case we apply a simple fuzzy modelling system to derive the PDOA, or they may be derived from self learning via clinical trials, in which case we apply a self-organising learning system [20].

Regarding the AER signals, it is considered that they relate to unconsciousness more closely than cardiovascular indicators. Hence, the first-level estimates of DOA from online AER signals are defined as the dominant depth of inaesthesia (DDOA). The MLAER has been validated as a measure of DOA. Conventional averaging and autoregressive model (ARX) techniques are used to smooth the MLAER and improve the signal to noise ratio (S/N) [27]. feature extraction is implemented to monitor the factors describing the changes in amplitudes and latencies of the MLAER waves which are related to DOA. Three factors have been defined and fused together using fuzzy logic to create an index for DOA. This index is a number which describes the value of DOA every $30 \mathrm{~s}$, defined as DDOA in this paper. However, at this level, the rules cannot be obtained from anaesthetists' experience because they have tittle experience in this monitoring mode. Hence, a selforganising learning system derived from a SOFM algorithm has been used to learn the rule base from offline data [28].

Using the previous methods for measuring SAP and HR to monitor PDOA and for measuring AER to monitor DDOA, we fuse these two factors to decide the first level of depth of anaesthesia (FDOA). There are three states of anaesthesia for FDOA i.e. anaesthetic light (AL), anaesthetic $O K(\mathrm{AO})$ and anaesthetic deep (AD). From extensive discussion with anaesthetists, a rule base to decide FDOA has been elicited, as shown in Table 1 .

Table 1: Anesthetists, rule base for FDOA

\begin{tabular}{llll}
\hline PDOA & \multicolumn{2}{l}{ DDOA (AER) } & \\
\cline { 2 - 4 } (SAP \& HR) & AL & AO & AD \\
\hline$A L$ & $A L$ & $A L$ & \\
$A O$ & $A L$ & $A O$ & $A D$ \\
$A D$ & & $A D$ & $A D$ \\
\hline
\end{tabular}

For notation see beginning of paper

IEE Proc.-Control Theory Appl., Vol. 146, No. 3, May 1999

\subsection{Second level of depth of anaesthesia (SDOA) from SW, LA and PR}

The second level to decide DOA is focused on measuring clinical signs (i.e. SW, LA and PR), for which there are no automatic methods of measurement as indicated in Fig. 1. The best way is to use qualitative concepts to identify this information. A scoring system is often used in this field. Thus fuzzy logic applied to the Apgar scoring system has been described by Shono et al. [29] in Japan. Similarly, we use fuzzy logic to determine the DOA from the qualitative clinical signs. The details of determining the degree of lightness (DOL) have been reported in Linkens et al. [20] and use a sum of scores obtained from SW, LA and PR indications. Basically, the SDOA is equal to DOL as shown in Table 2, while CDOA (confidence of DOA) is evaluated as (1-DOL).

\subsection{Fusing FDOA and SDOA to decide DOA}

Using the previous first level of measuring SAP, HR and AER to decide FDOA and the second level to decide SDOA, we fuse the first and second levels to decide DOA and provide a measure of confidence of depth of anaesthesia (CDOA). The rules for fusing the first and second levels are as follows:

IF $\mathrm{FDOA}=\mathrm{AD}$ THEN $\mathrm{DOA}=\mathrm{AD}$ and $\mathrm{CDOA}=100 \%$ IF $\mathrm{FDOA}=\mathrm{AO}$ THEN DOA $=\mathrm{AO}$ and $\mathrm{CDOA}=100 \%$

IF $\mathrm{FDOA}=\mathrm{AL}$ THEN go to second level as shown in Table 2 .

There are three states of DOA for anaesthetic light i.e. small light (SL), medium light (ML) and very light (VL) from Table 2. It depends on the sum of scores of sweating, lacrimation and pupil response. The confidence in DOA in Table 2 can provide an assessment of the monitoring of DOA using clinical signs. Anaesthetists are particularly concerned about the patients becoming 'light' during an operation. From the point of view of controlling a drug the infusion rate of the administered drug will be kept increasing when the patient is in a light situation. Therefore if these additional clinical signs cannot actually be used to represent $\mathrm{DOA}$ for this condition, too much drug will be given. Hence, if the patient is in a very light situation, anaesthetists will lose their confidence in this method to monitor DOA. Because patients vary a great deal, anaesthetists depend on their clinical experience to select monitoring parameters to control a drug. However, something may still happen which anaesthetists had not anticipated before the operation. Therefore the CDOA in this method

Table 2: Monitoring and assessment of DOA by second level

\begin{tabular}{llllll}
\hline No. & Sum & DOL $(\%)$ & SDOA (\%) & DOA & CDOA (\%) \\
\hline 1 & 0 & 16 & 16 & SL & 84 \\
2 & 1 & 25 & 25 & SL & 75 \\
3 & 2 & 25 & 25 & SL & 75 \\
4 & 2 & 50 & 50 & $\mathrm{ML}$ & 50 \\
5 & 3 & 50 & 50 & $\mathrm{ML}$ & 50 \\
6 & 3 & 50 & 50 & $\mathrm{ML}$ & 50 \\
7 & 4 & 50 & 50 & $\mathrm{ML}$ & 50 \\
8 & 4 & 75 & 75 & $\mathrm{VL}$ & 25 \\
9 & 5 & 75 & 75 & $\mathrm{VL}$ & 25 \\
10 & 6 & 100 & 100 & $\mathrm{VL}$ & 0 \\
\hline
\end{tabular}

For notation see beginning of paper 
gives an indicator which can warn and assist anaesthetists in the operating theatre for special cases.

\section{Multiechelon system of fuzzy-based support system for anaesthesia monitoring and control}

From the details given in the previous Section we propose a multistage hierarchical fuzzy control system with a multiechelon structure for anaesthesia as shown in Fig. 2. According to this hierarchical architecture there are four echelons which monitor, control, interpret and assess the whole surgical operation. Echelon 1 is a measurement and control action level which involves instrument sensors (Dinamap and AER equipment), anaesthetist observations (measurement of SW, LA and PR) and a syringe infusion pump (Graseby 3400 pump). Echelon 2 is an interpretation level which involves interpreting SAP and HR to provide PDOA, interpreting AER signals to give DDOA and interpreting SW, LA and PR to provide SDOA (i.e. DOL). Echelon 3 is a regulation level which involves controlling the drug from the anaesthetists' rule base (linguistic controller LC) or a SOFLC algorithm, and managing alarm situations. Finally, echelon 4 is an assessment level which assesses the whole surgical procedure according to DOA and confidence in DOA.

\section{Simulator for hierarchical fuzzy-based support system for anaesthesia monitoring and control}

A fuzzy logic based simulator programme called SADA (simulator advisor for depth of anaesthesia) has been programmed in "Modula-2" [30]. SADA has been developed to simulate the administration of intravenous and analgesic drugs. It fuses online measurements (such as systolic arterial pressure SAP and heart rate HR) and nonnumerical clinical signs (such as sweating, lacrimation and pupil response) using anaesthetists' experience or selforganising fuzzy logic control (SOFLC) algorithms to provide advice on administering drugs to a patient. This hierarchical control architecture includes five sectors: monitoring depth of anaesthesia, drug controller algorithm, deciding sensitivity of the patient, fentanyl supplementation advice, and recovery time calculation. It has been

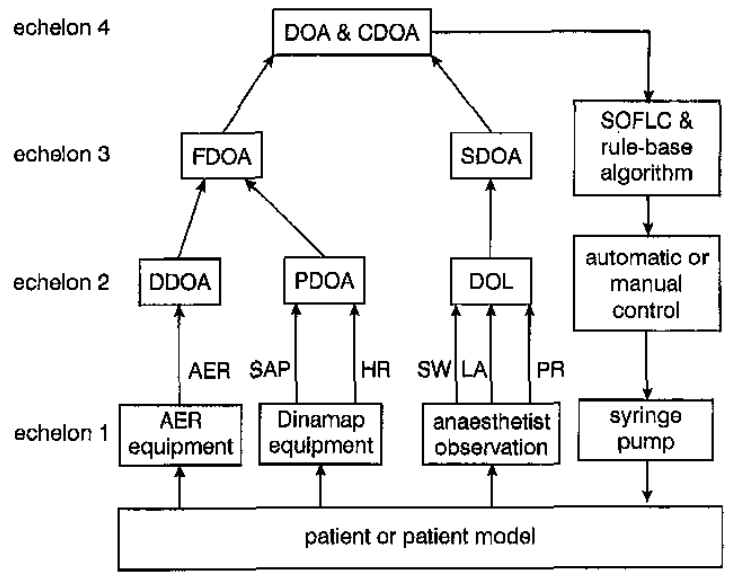

Fig. 2 Multichelon system for monitoring and controlling $D O A$ For notation see beginning of paper developed to predict drug profiles, control drug levels, and assess recovery time during anaesthesia. Also, a patient model has been designed according to linguistic rules and fuzzy set theory for the simulator describing dynamic changes during the induction and maintenance stages.

Moreover, a self-organising learning algorithm using offline analysis of patients' AER signals has been created from ten clinical trials during maintenance of anaesthesia using propofol [28]. Hence, the patient model with respect to not only SAP and HR but also AER has been merged into the system to simulate the whole procedure in the operating theatre. Therefore the computer screen of the simulator shows the fusing of SAP and HR to provide PDOA, interpreting AER signals to give DDOA, interpreting SW, LA and PR to provide SDOA, the fusing of PDOA and DDOA to provide FDOA, and the fusing of FDOA and SDOA to provide DOA and CDOA. Also, the controller based on SOFLC or LC is shown on the computer screen to determine the performance of the particular controller used by each simulation.

\section{Closed-loop control simulation and results}

The simulator has been used to simulate a major surgical procedure (abdominal operation), where the controller was based on either SOFLC or a linguistic controller (LC). In Fig. 3 the controller used a SOFLC algorithm which has a learning ability to adjust rules to suppress the surgical disturbances. Therefore when large disturbances (e.g. organ cutting) occurred to the patient, the SAP went high outside the acceptable band as shown in Fig. 3 so that the PDOA and DDOA went into the anaesthetic light range as shown in Figs. 4 and 5. The fusing of PDOA and DDOA to provide FDOA also indicated anaesthetic light as shown in Fig. 6. Further, the second level with SW, LA and PR went into the anaesthetic light range so that the SDOA (i.e. DOL) indicated small light degree as shown in Fig. 7 and confidence in the adequacy of control for the whole system was reduced from $100 \%$ to $75 \%$ as shown in Fig. 8 . The DOA was assessed as small light as shown in Fig. 9 and therefore the propofol infusion rate IR increased as shown in Fig. 10 to reduce SAP and increase PDOA and DDOA

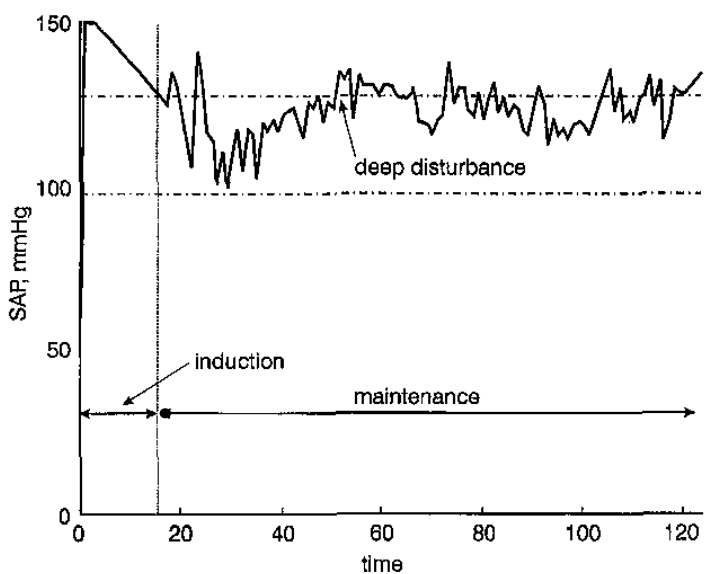

Fig. 3 Closed-loop simulation result, with patient model using SOFLC algorithm for systolic arterial pressure.

Arrows indicate time of starting maintenance phase and disturbance occurrence. Abscissa: 35 per unit in induction period, 1 min per unit in maintenance and recovery period 


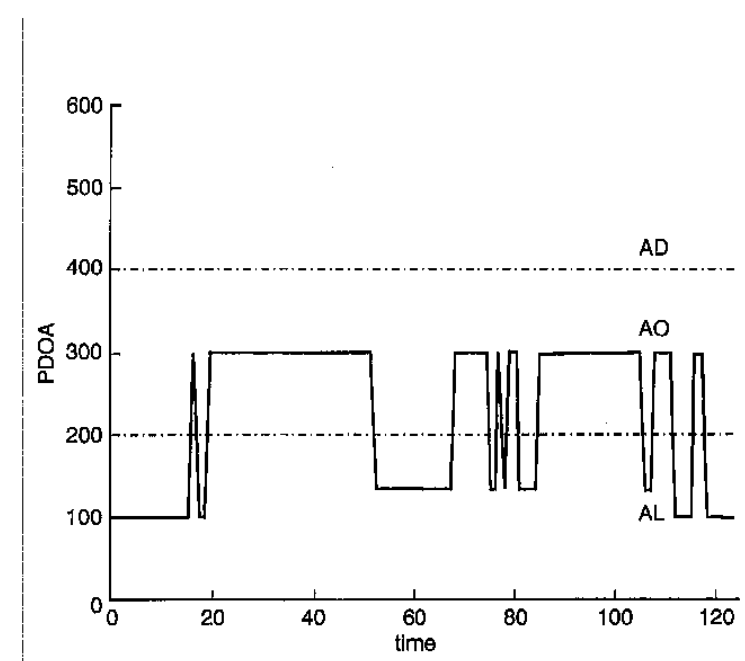

Fïg. 4 Primary-level DOA for Fig. 3 situation

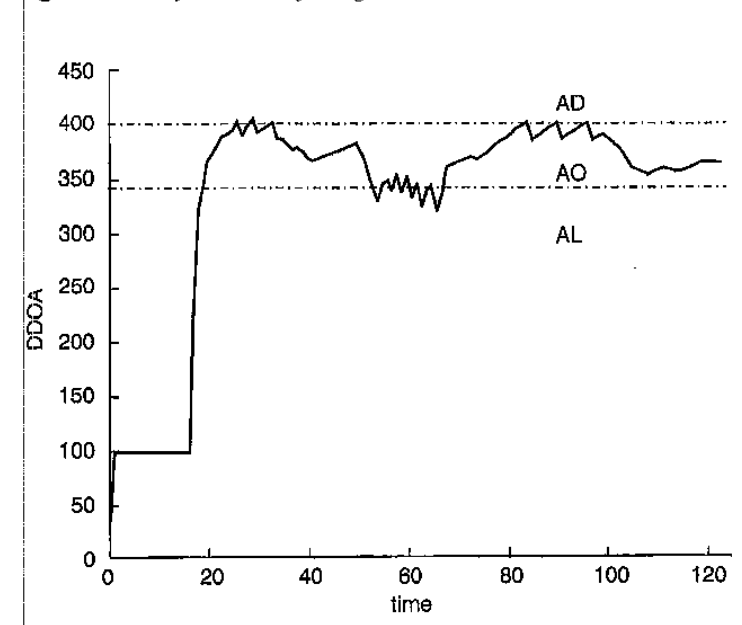

Fig. 5 Dominant-level DOA for Fig. 3 situation

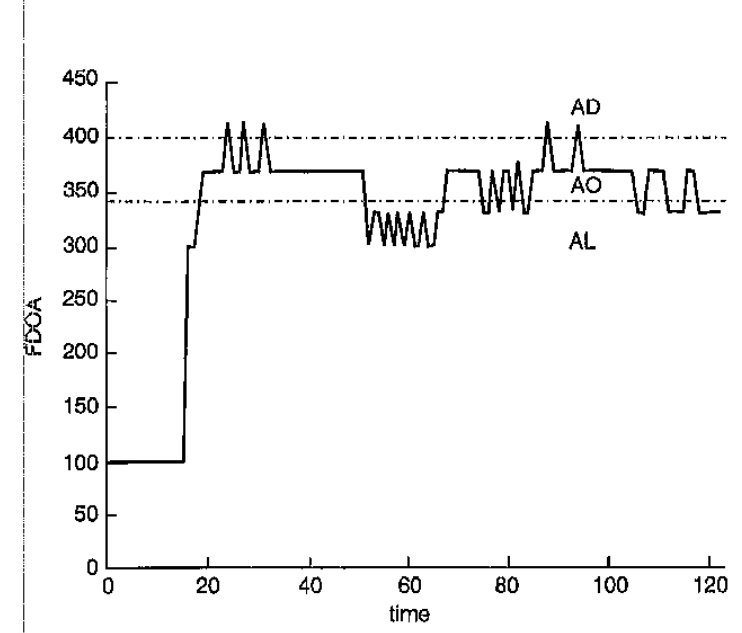

Fig. 6 First-level DOA for Fig. 3 situation

back to the anaesthetic $O K$ range. Hence, the SDOA returned to normal and confidence was increased to $100 \%$. Further, the DOA was returned to the anaesthetic $O K$ range.

In Figs. 11-18 the controller used an LC controller whose rules came from anaesthetists' experience in clinical practice. The trend in Figs. 11-18 was quite similar to that in Figs. 3-10. However, the standard deviations of SAP and DDOA as shown in Figs. 3 and 5 were 7.93 and 20.34,

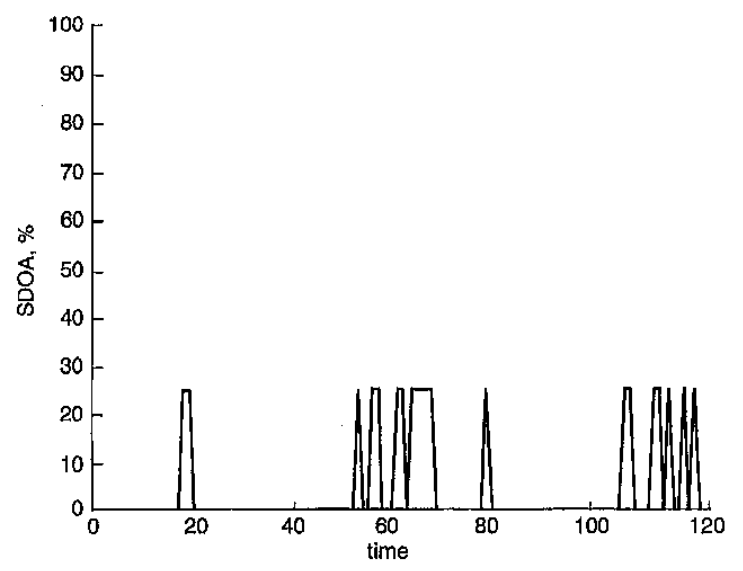

Fig. 7 Secondary-level DOA for Fig. 3 situation

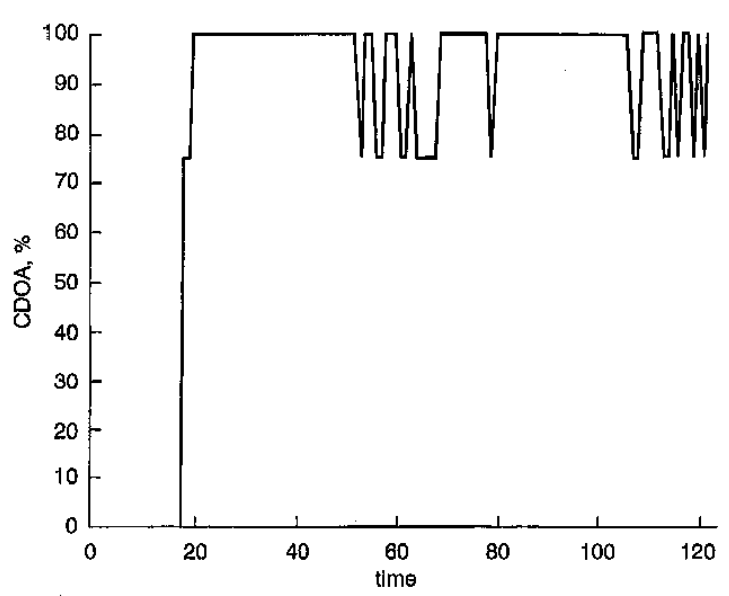

Fig. 8 Confidence in DOA for Fig. 3 situation

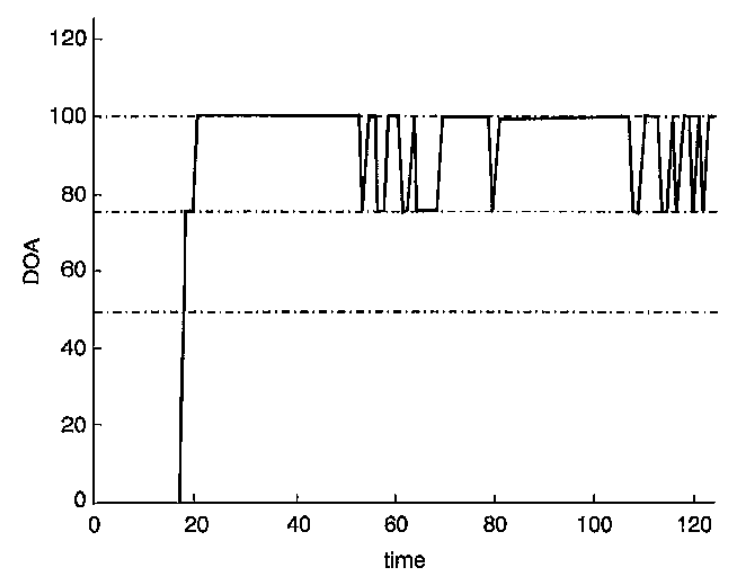

Fig. 9 Depth of anaesthesia for Fig. 3 situation

respectively, compared to 13.90 and 24.37 in Figs. 11 and 13. This indicates that the drug administered by the SOFLC showed a smoother time profile to the disturbances compared with the LC. Also, the percentage of time showing no degree of lightness was $82.2 \%$ in Fig. 7 in comparison to $73.3 \%$ in Fig. 15 during the maintenance phase. Therefore the monitoring and control confidence 


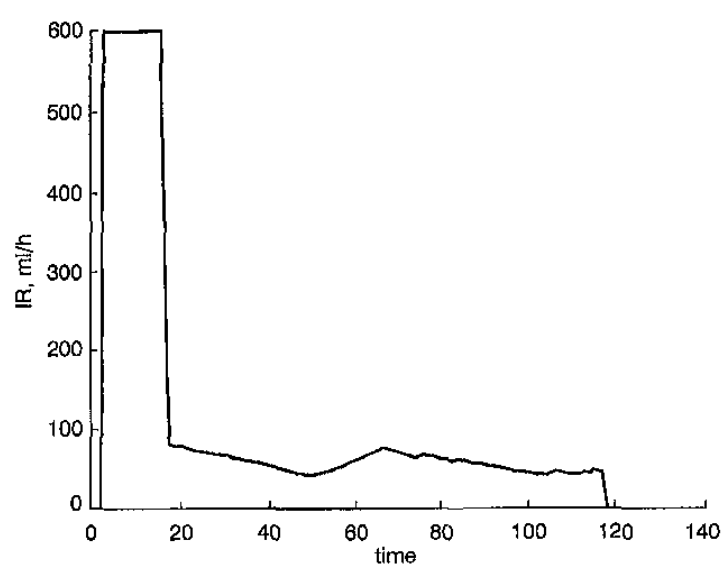

Fig. 10 Infusion rate for Fig. 3 situation

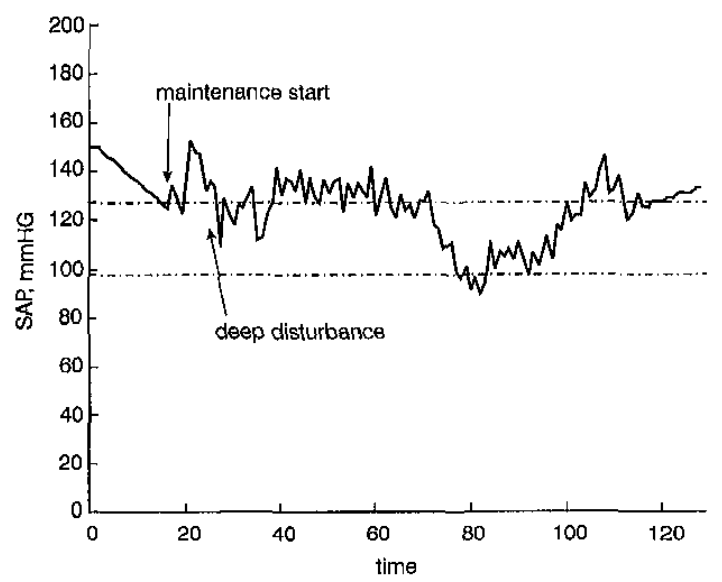

Fig. 11 Closed-loop simulation result, with patient model using controller Arrow linguistic and abscissa as for Fig. 3

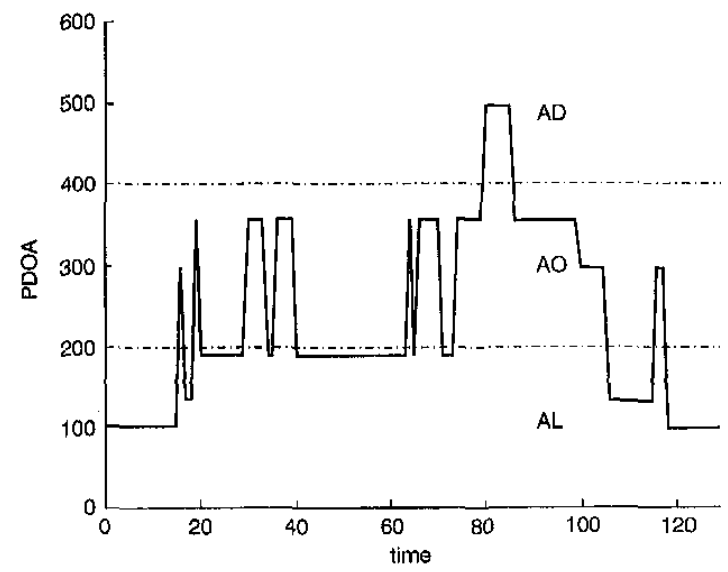

Fig. 12 Primary-level DOA for Fig. II situation

achieved when using SOFLC was better than when using LC. Moreover, the total drug administered during the whole simulation from induction to recovery stage was $103 \mathrm{ml}$ in Fig. 10 in comparison to $119 \mathrm{ml}$ in Fig. 18 which shows that the SOFLC controller can also provide reduced drug consumption.

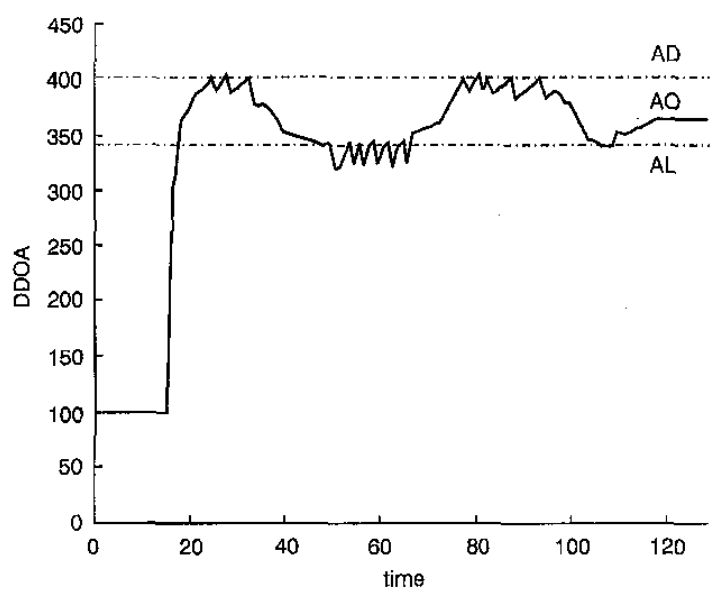

Fig. 13 Dominant-level DOA for Fig. II situation

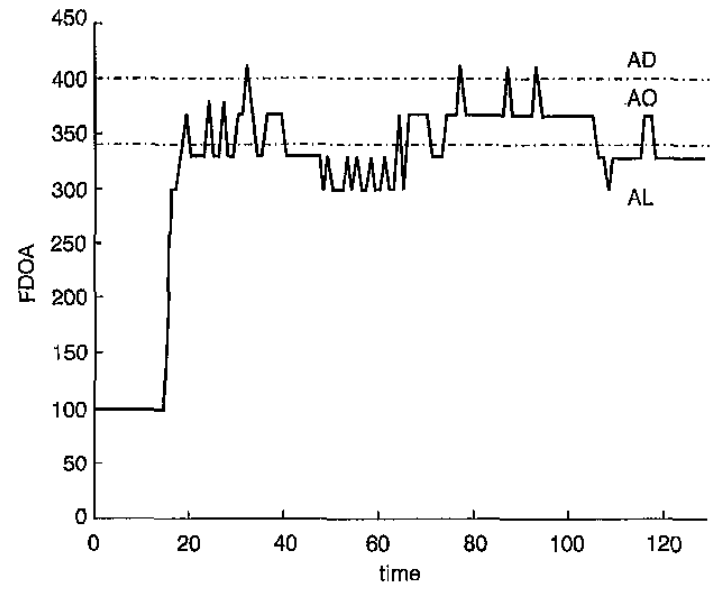

Fig. 14 First-level DOA for Fig. 11 situation

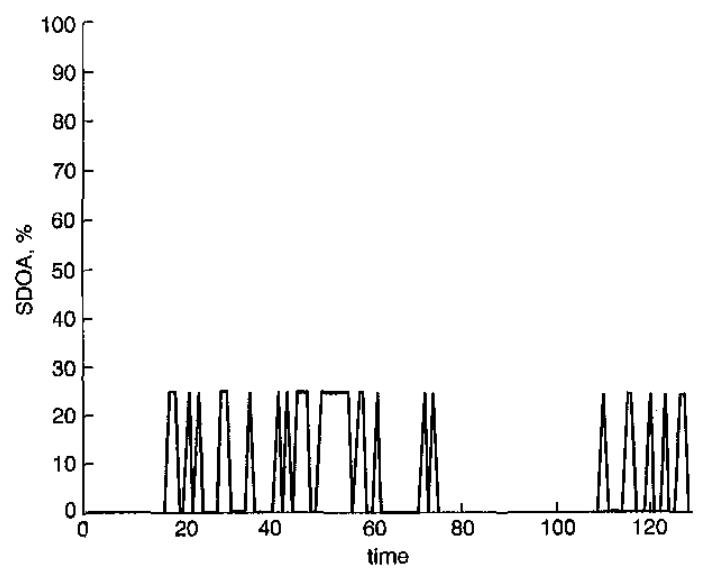

Fig. 15 Secondary-level DOA for Fig. 11 situation

\section{Conclusions}

Depth of anaesthesia is hard to define and so cannot depend merely on one physiological signal. Blood pressure depends on many factors such as blood volume and cardiovascular function so that it is not directly coupled

IEE Proc.-Control Theory Appl., Vol. 146, No. 3, May 1999 


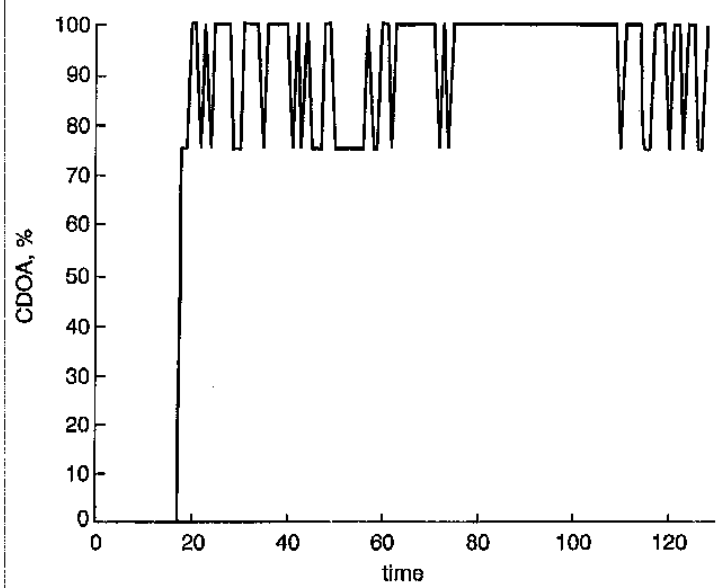

Fig. 16 Confidence in DOA for Fig. 11 situation

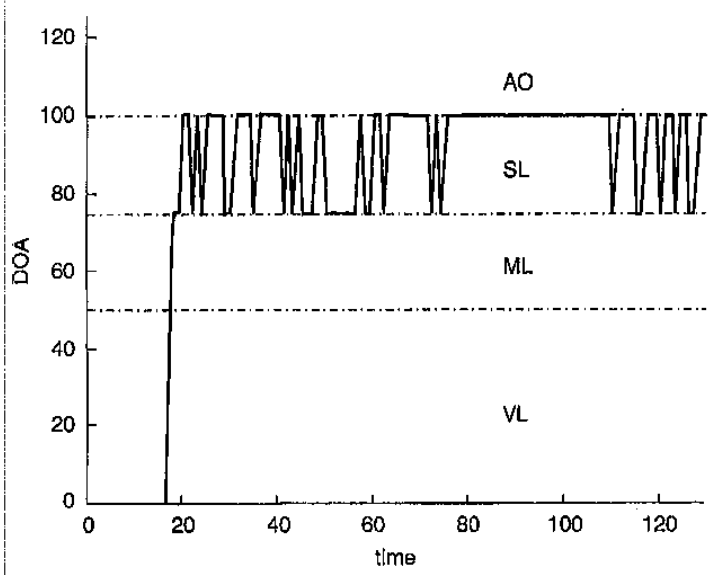

Fiig. 17 Depth of anaesthesia for Fig. 11 situation

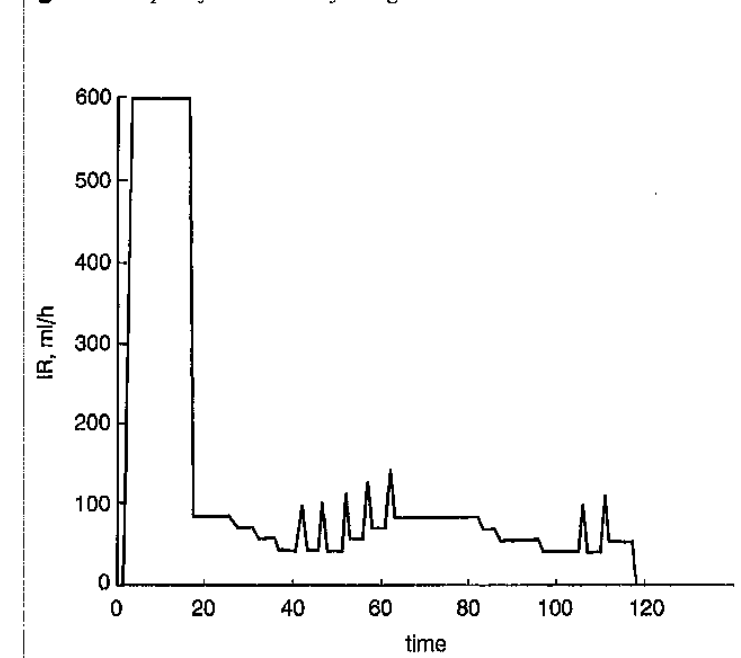

Fig. 18 Infusion rate for Fig, 11 situation

to anaesthetic depth. Also, the body function signs, such as SW, LA and PR, are too subjective and cannot be used for online measurement to the system. The AER is considered to be a better indicator of the DOA. However, AER signals ryquire a lot of signal processing and are easily contaminated by noise, such as diathermy effects.

ILE Proc.-Control Theory Appl., Vol. 146, No. 3, May 1999
Hence, a hierarchical fuzzy-based support system for anaesthesia monitoring and control by using AER signals for augmenting both cardiovascular (i.e. SAP and HR) and body function (i.e. SW, LA and PR) signs has been developed and used to control major surgical operations. This method of fusing multiple clinical signs to determine the DOA is similar to anaesthetists' clinical practice. Also, it has been demonstrated successfully via simulation that administration of intravenous anaesthetic drugs via fusing these multiple signs as feedback signals compared to just one signal [28] produces better procedures. Regarding the type of controller used, the SOFLC algorithm which has a learning ability to adjust rules to suppress the surgical disturbances has demonstrated a smoother response to the disturbances, more confidence in the whole system performance and less drug consumption compared to the LC.

Following extensive simulation studies, the next phase involves the implementation of these techniques for advisory-based control of DOA in operation theatre for intravenous anaesthesiology. The real-time measurement system is based on fuzzy logic, with the addition of fuzzy and multiresolution wavelets analysis signal processing of the AER signal [31]. The measuring system includes a signal processing chip hosted in a PC because of the high-speed, complex algorithm requirements providing averaging and analysis using multiresolution wavelets analysis. The analysed signal is fed to the neurofuzzy system where the inference takes place to obtain a measure of the DOA. Another measure for DOA is based on cardiovascular system status using a rule-based fuzzy logic classifier. The two measures are merged together using rule-based fuzzy logic data fusion to decide the final DOA. Based on the classified DOA, a target concentration is decided by a rule-based fuzzy logic controller which feeds the target to the increasingly-accepted anaesthetic procedure based on target controlled infusion. The system formed a closed-loop controller for monitoring and controlling the DOA for patients undergoing surgical operations, and is currently undergoing clinical validation at the Royal Hallamshire Hospital, Sheffield. Initial results have been encouraging, evidencing good clinical procedures using the system. Considerable experience already exists for evaluating these types of system (albeit simpler) in operating theatre for both inhalational and intravenous anaesthesia [32].

The simulator program is being developed further for the purpose of training junior anaesthetists, particularly for handling critical incidents. Several linguistic and numeric patients models have been developed for this purpose and incorporated into a self-contained anaesthetic training simulator, which encapsulates the architecture described in this paper [33].

\section{Acknowledgments}

The project has been supported financially by EPSRC under grant number GR/K08345.

\section{References}

1 MESAROVIC, M. D., MACKO, D., and TAKAHARA, Y.: 'Theory of hierarchical, multilevel system' (Academic press, New York, 1970)

2 HARAKAWA, T, and KAWAGUCHI, T.: 'Digital control in iron and steelmaking processes', Automatica, 1993, 29, (5), pp. 1185-1202

3 GANGULY, S., and SARAF, D. N.: 'On-line optimization: a hierarchical scheme for distillation column control', Chem. Eng. Process., 1992, 31, scheme for distillation column control', Chem. Eng. Process, , 1992, 31,
(6), pp. 337-347

4 LIANG, C. C.: 'An overview of the hierarchical dispatch and control system in Taipower', IEEE Trans. 1990, PWRS-5, (4), pp. 1041-1046 
5 RUBAAI, A.: "Transient stability control - a multi-level hierarchical approach', IEEE Trans. 1991, PWRS-6, (1), pp. 262-268

6 NADIRA, R., DY LIACCO, T. E., and LOPARO, K. A.: 'A hierarchica interactive approach to electric power system restoration', IEEE Trans. 1992, PWRS-7, (3), pp. 1123-1131.

7 HAIMES, Y. Y., TARVAINEN, K., SHIMA, T., and THADATHIL, J. 'Hierarchical multiobjective analysis of large-scale systems' (Hemisphere Publishing, New York, 1990)

8 LI, D., and HAIMES, Y. Y.: 'Multilevel methodology for a class of nonseparable optimization problems', Int. J. Syst. Sci., 1990, 21, (11), pp. $2352-2360$

9 LI, D.: 'Hierarchical control for large-scale systems with general multiple linear-quadratic structure', Automatica., 1993, 29, (6), pp. 14511461

10 JIA, L. M., and ZHANG, X. D.: 'On fuzzy multiobjective optimal control', Eng. Appl. Artif. Intell, 1993, 6, (2), pp. 153-164

11 ZADEH, L. A.: 'Fuzzy sets', Inf. control, 1965, 8, pp. 28-44

12 MAMDANI, E. H.: "Application of fuzzy algorithms for control of simple dynamic plant', Proc. IEEE, 1974, 121, pp. 1585-1588.

13 SUGENO, M., (editor) 'Industrial applications of fuzzy control' (North Holland, Amsterdam, 1985).

14. HIROTA, K., and SUGENO, M.: 'Industrial applications of fuzzy technology in the world' in 'Advances in fuzzy systems: applications and theory' (World Scientific, Singapore, 1985 , vol. 2 ).

15 LINKENS, D. A., and MAHFOUF, M.: 'Fuzzy logic knowledge-based control for muscle relaxant anaesthesiology'. Proceedings of IFAC symposium 'Modelling and control in medicine', 1988, Venice, pp. 185-190

16 YING, H., SHEPPARD, L., and TUCKER, D. 'Expert-system-based fuzzy control of arterial pressure by drug infusion', Med. Prog through Technol. $1988, \mathbf{1 3}, \mathrm{pp} .203-215$

17 MEIER, R., NIEUWLAND, J., ZBINDEN, A. M., and HACISALIHZADE, S. S.: 'Fuzzy logic control of blood pressure during anaesthesia', IEEE Control Syst. Mag., 1992, 12, (12), 12-17

18 TSUTSUI, T., and ARITA, S.: 'Fuzzy-logic control of blood pressure through enflurane anesthesia', J. Clin. Monit., 1994, 10, 110-117

19 ZBINDEN, A. M. FEIGENWINTER, P. PETERSEN-FELIX, S, and HACISALIHZADE, S.: 'Arterial pressure control with isoflurane using fuzzy logic', British J. Anaesth., 1995, 74, pp. 66-72.

20 LINKENS, D. A., SHIEH, J. S., and PEACOCK, J. E.: 'Hierarchical fuzzy modelling for monitoring depth of anaesthesia, Fuzzy Sets Syst., 1996,79 , pp. $43-57$

21 BUHRER, M., MAITRE, P. O, CREVOISIER, C., and STANSKI, D. R.: 'EEG effects of benzodiazepines. 1 . Choosing an EEG parameter to measure the effect of midazolam on the central nervous system' Clin. Pharmacol. Ther, 48, (5), 1990, pp. 544-553.

22 STANSKI, D. R.: "Pharmacodynamic modelling of anaesthetic EEG drug effects', Annu. Rev. Pharmacol. Toxicol,, 1992, pp. 423447.

23 HOFFMAN, W. E., and EDELMAN, G.: 'Comparison of isoflurane and desflurane anaesthetic depth using burst suppression of the EEG in neurosurgical patients', Anaesth. Analg., 1995, pp. 811-816.

24 BARNETT, T. P., JOHNSON, L. C., NIATOH, P. HICKS, N., and NUTE, C.: 'Bispectrum analysis of EEG signals during waking and sleeping', Science, 172, 1971, pp. 401-402.

25 THORTON, C., CREAGH-BARRY, P., JORDAN, C., and NEWTON, D. E. F.: 'Anaesthetic depth and auditory evoked potentials', Acta. Anaesth. Scand. 1993, 37, (S100), pp. 10S-108.

26 SCHWENDER, D., MADLER, C., KLASING, S., PETER, K, and POPPEL, E.: 'Do auditory cvoked potentials measure depth of anaesthesiology ?', Theor. Surg., 1993, 8, pp. 29-37.

27 ELKFAFI, M., SHIEH, J. S., LINKENS, D. A., and PEACOCK, J. E.: 'Intelligent signal processing of evoked potentials for anaesthesia monitoring and control', IEE Proc. Control Theory Appl., 1997, 144, (4), pp. 354-360

28 ELKFAFI, M SHIEH, J S LINKENS, D. A., and PEACOCK, J. E.: 'Fuzzy logic for auditory evoked response monitoring and control of depth of anaesthesia', Fuzzy Sets Syst., 1998, 1000, pp. 29-43.

29 SHONO, H., OGA, M., SHIMOMURA, K., YAMASAKI, M., ITO, Y., MURO, M., and SUGIMORI, H.: "Application of fuzzy logic to the apgar scoring system', Int. J. Biomed. Comput, 1992, 30, pp. 113-123

30 LINKENS, D. A., SHIEH, J. S., and PEACOCK, J. E.: "SADAP: simulator for hierarchical fuzzy control of depth of anaesthesiology'. Proceedings of NAFIPS/IFIS/NASA' conference, December 1994, San Antonio, pp. 386-390.

31 LINKENS, D. A., ABBOD, M. F., and BACKORY, J. K.: "Auditory evoked responses for measuring depth of anaesthesia using wavelet fuzzy logic system', Proceedings of IFAC symposium on 'Modelling and control in biomedicine systems', 23-26 March, 1997, Warwick, pp. 193198.

32 LINKENS, D. A., SHIEH, J. S., ELKFAFI, M., and PEACOCK, J. E: 'Hierarchical fuzzy-based support system for anaesthesiology monitoring and control'. Presented at the IEEE international conference on Fuzzy Systems, 8-11, Sep.1996, New Orleans, USA

33 ABBOD, M. F., and LINKENS, D. A.: 'Anaesthesia simulators: intelligent monitoring and control of depth of anaesthesia'. Presented at the IEE colloquium on Simulation in Medicine, Dec., 1998, Savoy Place, London. 